\title{
Surgical and functional outcome of sigmoid colon-vaginoplasty in Mayer-Rokitansky-Kuster-Hauser syndrome
}

\section{Pradyumna Pan*}

Department of Pediatric and Reconstructive Surgery Unit, Ashish Hospital and Research Centre, Jabalpur, Madhya Pradesh, India

Received: 22 July 2017

Accepted: 22 August 2017

\section{*Correspondence:}

Dr. Pradyumna Pan,

E-mail: dr_pan@rediffmail.com

Copyright: (c) the author(s), publisher and licensee Medip Academy. This is an open-access article distributed under the terms of the Creative Commons Attribution Non-Commercial License, which permits unrestricted non-commercial use, distribution, and reproduction in any medium, provided the original work is properly cited.

\section{ABSTRACT}

Background: The objective of this study is to report our experience with sigmoid vaginoplasty in adolescent female patients of Mayer-Rokitansky-Kuster-Hauser (MRKH) syndrome with emphasis on the effectiveness of surgery, complications, sexual and functional outcomes.

Methods: A retrospective study of adolescent females with vaginal atresia and Mayer-Rokitansky-Kuster-Hauser syndrome. The sigmoid segment was used for vaginoplasty in all the case.

Results: Eleven females were studied over a period of 15 years. Postoperative complications were perineal rash in 3 , ileus in 3, and minor wound infection in 1 patient and urinary tract infection in one patient. Nine patients were on regular follow-up. All the neovaginas were patent and functional. The postoperative mean vaginal length was $12.1 \mathrm{~cm}$ (range $11 \mathrm{~cm}$ to $14 \mathrm{~cm}$ ). One of the patients had mild vaginal stenosis which responded to vaginal dilation, one had mucosal prolapse of the neovagina, one had excessive mucus discharge, during follow-up visits. Out of the 11patients, 8 patients are sexually active and 7 reported that they were engaging in satisfactory sexual activity with mild or no pain, and with good mucosal sensitivity. Only one experienced dyspareunia and three patients had not participated in sexual activity.

Conclusions: Sigmoid vaginoplasty is a safe and acceptable procedure for vaginal agenesis with good cosmetic results and acceptable complications rate. Sigmoid colon vaginoplasty is the treatment of choice because of its large lumen, thick walls resistant to trauma, adequate secretion allowing lubrication, not demanding prolonged dilatation and short recovery time.

Keywords: Mayer-Rokitansky-Kuster-Hauser syndrome, Vaginal agenesis, Vaginal reconstruction

\section{INTRODUCTION}

Congenital absence of the vagina is a relatively rare condition most commonly associated with MayerRokitansky-Kuster-Hauser (MRKH) syndrome with an incidence of one in 4000 to 5000 female newborn. The purpose of vaginal agenesis treatment is not only to establish an adequate passageway for penetration but also to facilitate satisfactory sexual intercourse. Historically, several reconstructive techniques have been described to provide for functional vaginal reconstruction on these patients, both operative and non-operative. These various procedures have its own peculiar advantages and disadvantages.

The Mayer-Rokitansky-Küster-Hauser (MRKH) syndrome is characterized by congenital aplasia of the uterus and the upper part (2/3) of the vagina in women 
showing normal development of secondary sexual characters, normal functional ovaries and a normal 46, XX karyotype. MRKH may be isolated (type I) but it is more frequently associated with renal, vertebral, and, to a lesser extent, auditory and cardiac defects (MRKH type II or MURCS association). The first sign of MRKH syndrome is a primary amenorrhea.

The use of isolated bowel segments has been shown to provide excellent results, circumventing the need for regular dilatation and providing natural lubrication. ${ }^{1} \mathrm{We}$ present our results of sigmoid vaginoplasty performed in patients with MRKH syndrome, with emphasis on the operative and sexual outcomes of these patients.

\section{METHODS}

This is a retrospective study from 2001 to 2016. All the 11 patients listed in the study were having MRKH syndrome. Six $(54.5 \%)$ presented with isolated uterovaginal aplasia (MRKH type I). In other five women two had renal agenesis, one had ectopic pelvic kidney, one with absent 7th and 8thrib and one had scoliosis. All the patients underwent sigmoid colon vaginoplasty under general anesthesia. Preoperative work up included physical examination, karyotyping, abdominal and pelvic ultrasonography, and endocrinological and psychological assessments. Informed consent, after an explanation of the potential benefits and risks of sigmoid vaginoplasty and the surgical or nonsurgical alternatives to create a neovagina was obtained from all the patients and parents. Three months after the procedure, patients were allowed to start intercourse. The patients were analysed for the effectiveness of surgery, complications and sexual outcome.

\section{Surgical technique}

The approach was abdominoperineal in all the patients (Figure 1).

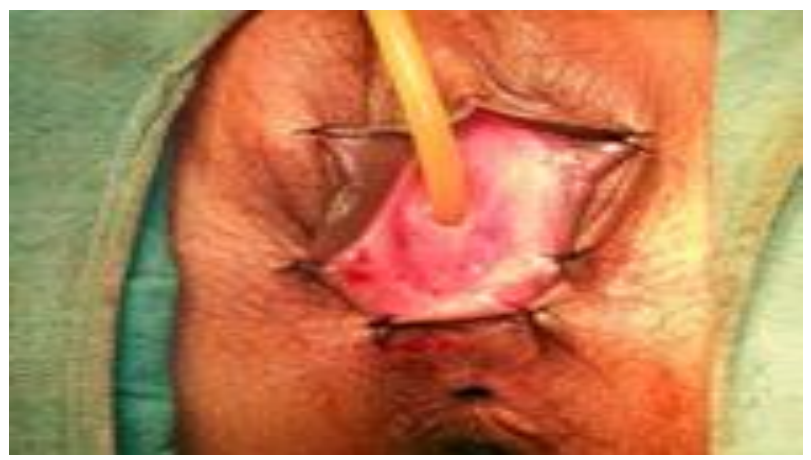

Figure 1: Preoperative showing absence of vagina.

Pfannenstiel incision was made to open the abdomen. After opening the abdomen, about $12-15 \mathrm{~cm}$ long segment of sigmoid colon was mobilized on its vascular pedicle (Figure 2). Vascular pedicle plane of the sigmoid graft was made in between the left colic and the superior rectal artery. The continuity of the colon was restored by a hand-sewn double-layer end-to-end anastomosis using 30 absorbable sutures. An isoperistaltic orientation was used. The proximal end of the sigmoid segment was closed in 2 layers and hitched to the sacral promontory with non-absorbable suture. Lower end of the graft was brought down to the perineum, after creating a rectovesical tunnel between urethra, bladder anteriorly, and rectum posteriorly (Figure 3, Figure 4). The edges of the vaginal pit were sutured to the distal end of the sigmoid segment. A Foley's catheter was kept in the bladder for 7 days. At discharge from the hospital, the patients were instructed to irrigate the neovagina daily for 8 weeks and weekly thereafter and vaginal introitus dilation twice daily for 2 months and thereafter once daily for 2 months.

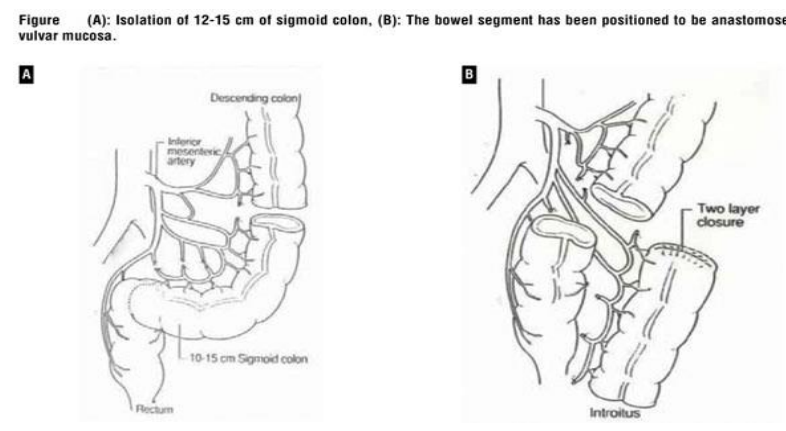

Figure 2: Line drawing showing creation of colon graft.

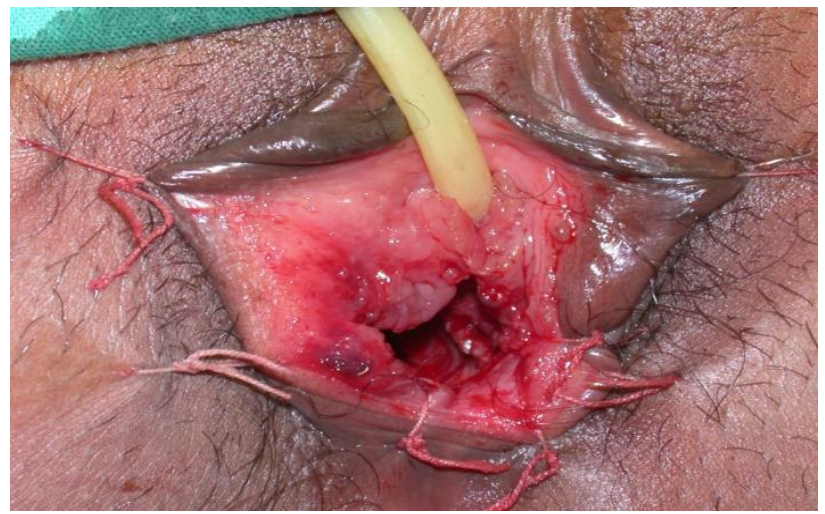

Figure 3: Space created for colon.

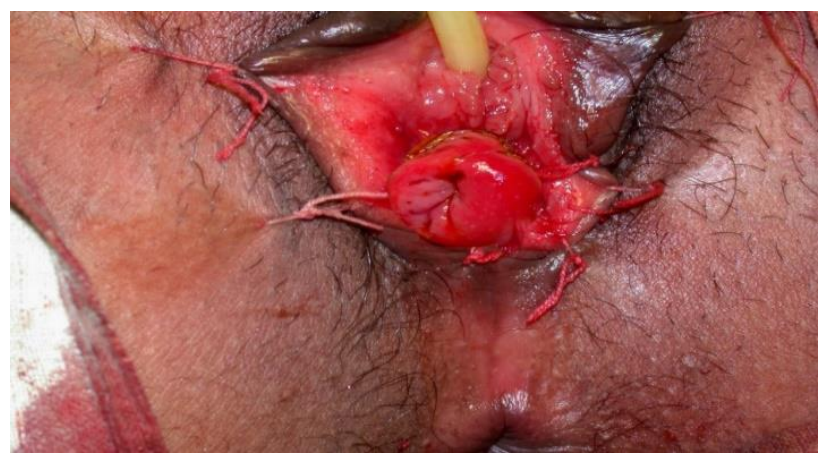

Figure 4: Pulled colon. 


\section{RESULTS}

The total duration of this study was 15 years. The total number of patients included in this study was 11 . The age range was 19-24 years (mean 21.1 years). All the patients presented with amenorrhea. The mean follow-up duration was 4.2 years. Two patients were lost to follow-up after 14 months and 22 months. None of the 11 patients had any significant per-operative complication. Three patients had mild postoperative ileus, managed conservatively. Three had transient perineal rash which disappeared after vaginal wash. One patient had a minor abdominal wound infection, which responded well to local wound care and systemic antibiotics. One patient had mild urinary tract infection.

Neovagina was cosmetically acceptable to the parents of all patients. One of the patients had mild vaginal stenosis during follow-up visits which responded to vaginal dilations. None of them had excessive mucus discharge. One patient had mucosal prolapse of the neovagina that required trimming.

All patients had normal external genitalia in appearance. The vagina was totally absent in six patients in the remaining 5 patients, vaginal pit ranged from $1 \mathrm{~cm}$ to 2 . $5 \mathrm{~cm}$.Ten patients had a total absence of the uterus and fallopian tubes. In one patient, a rudimentary uterus was observed. Ovaries were seen in all patients. Postoperative vaginal length varied from $11 \mathrm{~cm}$ to $14 \mathrm{~cm}$, mean $12.1 \mathrm{~cm}$. During the follow-up, no other complications, such as hemorrhages, infections were noted.

Out of these 11 patients, 8 are sexually active and 7 patients reported that they were engaging in satisfactory sexual activity with mild or no pain, and with good mucosal sensitivity (Figure 5); only one patient experienced severe pain and post coital bleeding during sexual intercourse.

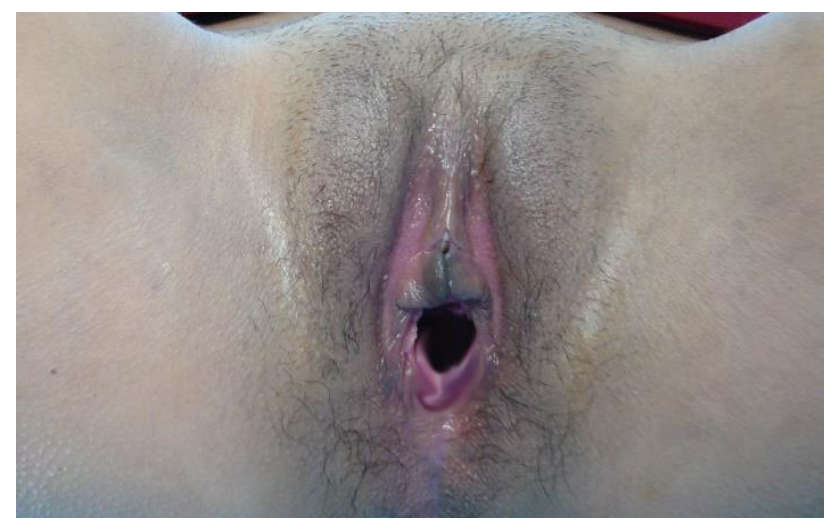

Figure 5: Postoperative after 6 months in sexually active woman.

Three did not engage in sexual intercourse, as they were not married (Figure 6). The patients were inquired how their accomplices encountered their recently made vaginas. Partner responses were presented as positive in 4 , neutral in 3 and negative in 1 . Three had good relation with partner, neutral in 4 and poor in 1 .

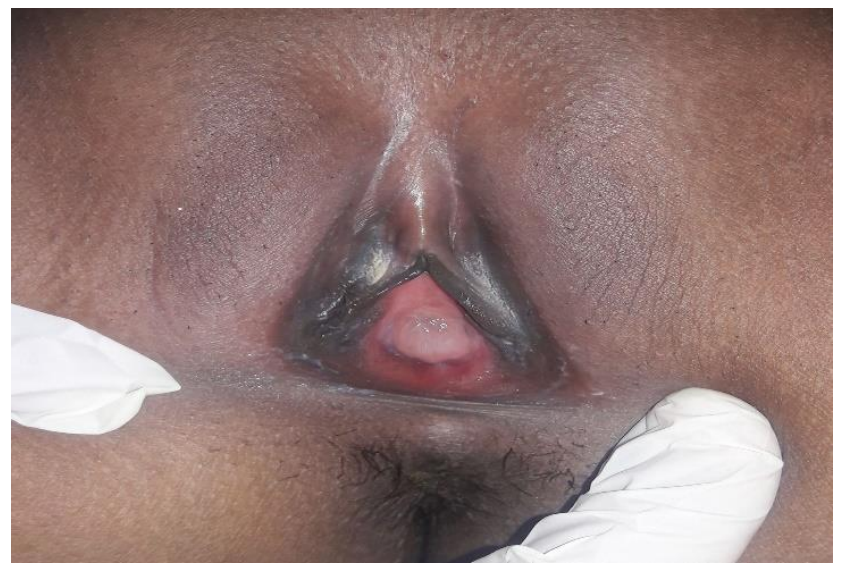

Figure 6: Postoperative after 6 months in sexually non-active woman.

\section{DISCUSSION}

Vaginal atresia due to Müllerian aplasia is noted in patients with MRKH syndrome. ${ }^{2}$ A young woman's sense of well-being and quality of life is crushed by this condition. Affected individuals without treatment will find it challenging, if not impossible, to engage in penilevaginal intercourse, they do not menstruate and they will be unable to carry a pregnancy. The finding that sexual intercourse will not occur without medical intervention and the awareness of absence of childbearing capacity may be devastating to an adolescent who has not yet reached certain developmental milestones.

Because of physical and psychological challenges, it has been suggested that the management of MRKH falls into two categories:

- $\quad$ the need to anatomically manage the anomaly so that young women may have the option to engage more easily in penile-vaginal intercourse and

- the need to help young women cope with the psychological impact of the condition. ${ }^{1}$

Probably the most important aspect with regard to reconstruction, especially in patients with MRKH, is the timing of the initial procedure. Ideally, the patient should be nearing the age for sexual intercourse, highly motivated, and emotionally mature. Patients who are relatively immature despite their chronological age are at great risk for poorer outcomes, which can lead to unfavourable healing. Failure of complete, unimpeded healing can result in the need for multiple revisionary procedures, the consequences of which may result in various degrees of sexual dysfunction. Thus, preoperative patient assessment and careful psychological preparation before any surgical intervention is crucial. 
In the past, vaginal reconstruction procedures were conducted on infants and pre-pubertal girls and this required inevitable surgical revision in adolescence before sexual activity. Deferring treatment allows the woman herself to be involved in decision making and also increases compliance with the adjuvant dilatation therapy that may be required. ${ }^{3}$

Diagnosis is frequently made clinically, but often confirmed either radiologically or laparoscopically, in patients whose hormonal and karyotype investigations for primary amenorrhea are normal. The use of ultrasonography, intravenous urography, computerized tomography, and magnetic resonance imaging provides information about the degree of abnormality and associated renal anomalies. ${ }^{4}$

Functional vaginal reconstruction must accomplish several essential requirements, adequate length of the vaginal wall, sufficient transverse dimensions of the introitus and vaginal pouch, healing without cicatricial contracture, the vaginal lining should be durable enough to withstand the forces associated with intercourse and should provide a satisfactory cosmetic result, leaving the external genitalia intact. ${ }^{5}$ All of these elements are essential to allow for ample and pain-free intercourse.

Treatment may be either surgical or nonsurgical. There are two main types of procedure. The first one consists of the creation of a new cavity and can be nonsurgical or surgical. The second is vaginal replacement with a preexisting canal lined with a mucous membrane (a segment of bowel). ${ }^{6}$ Commonly performed procedures are Franck's dilator method, Abbe-McIndoe operation, Vecchietti operation and Sigmoid colon vaginoplasty.

The traditional treatment for this condition is McIndoe technique which uses of split-thickness skin graft. ${ }^{7}$ This procedure has high rates of graft failure and stenosis. Frequent dilatations and need to wear a vaginal moulds at night are additional disadvantages. Incidence of dyspareunia directly correlates with the length of the neovagina, with an incidence of $100 \%$ if the vaginal length is less than $6 \mathrm{~cm} .{ }^{8}$ It is extremely difficult to have a good length of the vagina after few years of surgery and even after having a good length, vagina is dry and painful on coitus. ${ }^{8}$

Colon, cecum, or ileum may be used for bowel vaginoplasty but the sigmoid colon is preferred over the others. The sigmoid colon has certain advantages, such as, a thick wall, large diameter, does not traumatize easily, has adequate mucosal secretion, which although adequate for lubrication is not excessive or irritating, and does not require regular dilatation after the postoperative period. ${ }^{9}$ Also, it is close to the perineum, can easily be mobilized on vascular pedicle and it does not require moulds or stenting. ${ }^{10}$ Sigmoid vaginoplasty provides an esthetically pleasing neovagina with a good length, natural lubrication, and obviating the need for stenting and dilatation. ${ }^{10}$

In our series, no intra-operative or intestinal complications occurred. Intestinal complications are rare with sigmoid vaginoplasty and we recommend preoperative bowel preparation and meticulous suturing to avoid such complications.

Postoperative complications reported in the literature ranged from $0.2-1.4 \%$, noted are ileus, wound infection, pelvic hematoma, urinary tract infection, urinary incontinence, incision abscess, intestinal leakage, colorectal fistula, necrosis of the sigmoid conduit, pelvic abscess. ${ }^{11,12}$ In our series three had perineal rash induced by neovaginal discharge that disappeared after irrigation of the neovagina, mild ileus in 3 patients, one had a mild urinary tract infection and one had minor abdominal wound infection which responded to antibiotics.

Long-term complications noted in literature (0.2-13.9\%) are persistent vaginal discharge. stenosis of the neovagina, prolapse of the neovagina, malodor of the vaginal secretions, postcoital bleeding. ${ }^{13,14}$ None of the patients in our series had any major postoperative or chronic complications. None of the patients complained of local irritation and dryness. One woman had dyspareunia and post coital spotting. Excessive mucus discharge was reported by one patient in the initial months after surgery but subsided after 3-4 months. Thereafter, the patient experienced just a moist vagina with no complaint. ${ }^{15}$

Mucosal prolapse is part of the late postoperative complications that occurred in one of our patient, which was successfully managed by surgical excision of redundant tissue. Parsons et al. reported mucosal prolapse in $14 \%$ of patients. ${ }^{16}$ This can be prevented by selection of appropriate bowel segment length and proper fixation to the sacral promontory thus decreasing the incidence of this complication. It was made in all of our patients.

Stenosis of the neovagina is the main long-term complication reported $(8.8 \%)$ within the first postoperative year. However, we encountered 1 mild stenosis in our patients but fortunately she responded to regular vaginal dilations. The liability for anastomotic stricture can be decreased by selection of a bowel segment of adequate blood supply and length that can be mobilized and pulled easily to the perineum without tension, creation of a large enough space between bladder and rectum and generous anastomosis at the hymenal region. Several authors reported that patients were encouraged to use a vaginal dilator daily for a couple of weeks postoperatively, which may prevent stenosis of the neovagina and introitus. ${ }^{17} \mathrm{We}$ recommend regular postoperative dilatation of the anastomotic site till full sexual activity is chosen. 
Diversion colitis, ulcerative colitis is rare and has been reported in the neovagina. ${ }^{18,19}$ However, continuous follow-up of these patients with regard to these potential complications are necessary. On vaginal examination of our patients, we found that all patients had an outstanding cosmetic appearance of the neovagina with an adequate vaginal orifice.

Vaginal assessment revealed an adequate size of the neovagina in all patients. Patients were happy with their surgical outcome. Significant dyspareunia and bleeding during intercourse were reported in one. None of the patients complained of vaginal dryness or need for external lubricants but instead they experienced a moist vagina owing to the colonic mucosal secretion. ${ }^{20}$

The sexual satisfaction rate reported in the literature is approximately $80 \%$ to $90 \%$ With an assessment of the functional outcome among the 8 sexually active patients in our series, the sexual satisfaction rate was approximately $87.5 \%$ ( 7 of 8 patients) Partners experience towards newly created vaginas were positive in 4 , neutral in 3 and negative in 1 . Three had good relation with partner, neutral in 4 and poor in 1 resulting in separation and divorce.

Families of 3 patients were counselled to marry their daughters to widowers who had already completed their families or to men who were physically challenged and wanted to adopt children.

Although postsurgical results are acceptable to the parents and patients cosmetically, the long-term sexual and psychological outcomes are yet to be assessed. ${ }^{21}$ Most of these patients have functioning ovaries, which would therefore allow for in-vitro procedures through the use of a female surrogate which remains to be explored in the future.

\section{CONCLUSION}

Our study revealed that sigmoid vaginoplasty can provide the patient with a self-lubricating, aesthetically pleasing neovagina of adequate size. It has a low complication rate and a low incidence of introital stenosis with no need for daily vaginal dilatation or vaginal stenting by vaginal moulds. Moreover, the reported functional outcome is excellent.

\section{Funding: No funding sources}

Conflict of interest: None declared

Ethical approval: The study was approved by the Institutional Ethics Committee

\section{REFERENCES}

1. Hendren WH, Atala A. Use of bowel for vaginal reconstruction. J Urol. 1994;152:752-5.
2. Hughes IA. Disorders of sex development: a new definition and classification. Best Pract. Res Clin Endocrinol Metab. 2008;22:119-34.

3. Leduc B, van Campenhout J, Simard R. Congenital absence of the vagina. Observations on 25 cases. Am J Obstet Gynecol. 1968;100:512 -20.

4. Folch M, Pigem I, Konje JC. Mullerian agenesis: etiology, diagnosis, and management. Obstet Gynecol Surv. 2000;55:644- 9.

5. Hensle TW, Chang DT. Vaginal reconstruction. Urol Clin North Am. 1999;26:39 -47.

6. Gupta NP, Ansari MS. Mayer-Rokitansky-KusterHauser (MRKH) syndrome - a review. Indian J Urol. 2002;18:111-6.

7. ACOG Committee Opinion No. 355. Vaginal agenesis, diagnosis, management, and routine care. Obstet Gynecol. 2006;108:1605-9

8. De Souza AZ, Maluf M, Perin PM, Maluf Filho F, Perin LF. Surgical treatment of congenital uterovaginal agenesis: Mayer-Rokitansky-KusterHauser syndrome. Int Surg.1987;72:45-7.

9. Mungadi IA, Ahmad Y, Yunusa GH, Agwu NP, Ismail S. Mayer-Rokitansky-Kuster-Hauser Syndrome: Surgical Managementof Two Cases. J Surg Tech Case Rep. 2010;2(1):39-43.

10. Rawat J, Ahmed I, Pandey A, Khan TR, Singh S, Wakhlu A, Kureel SN. Vaginal agenesis: Experience with sigmoid colon neovaginoplasty. J Indian Assoc Pediatr Surg. 2010;15(1):19-22.

11. Imparato E, Alfei A, Aspesi G, Meus AL, Spinillo A. Long-term results of sigmoid vaginoplasty in a consecutive series of 62 patients. Int Urogynecol J Pelvic Floor Dysfunct. 2007;18(12):1465-9.

12. Kapoor R, Sharma DK, Singh KJ, Suri A, Singh P, Chaudhary H, Dubey D, Mandhani A. Sigmoid vaginoplasty: long-term results. Urology. 2006;67(6):1212-5.

13. Djordjevic ML, Stanojevic DS, Bizic MR. Rectosigmoid vaginoplasty: clinical experience and outcomes in 86 cases. J Sex Med. 2011;8(12):348794.

14. Nowier A, Esmat M, Hamza RT. Surgical and functional outcomes of sigmoid vaginoplasty among patients with variants of disorders of sex development. Int. Braz j urol. 2012;38:380-6.

15. Hanna MK. Vaginal construction. Urology. 1987;29:272-5.

16. Parsons JK, Gearhart SL, Gearhart JP. Vaginal reconstruction utilizing sigmoid colon: Complications and long-term results. J Pediatr Surg. 2002;37:629-33

17. Khen-Dunlop N, Lortat-Jacob $\mathrm{S}$, Thibaud E, Clément-Ziza $\mathrm{M}$, Lyonnet $\mathrm{S}$, Nihoul-Fekete $\mathrm{C}$. Rokitansky syndrome: Clinical experience and results of sigmoid vaginoplasty in 23 young girls. $\mathbf{J}$ Urol. 2007; 177:1107-11.

18. Froese DP, Haggitt RC, Friend WG. Ulcerative colitis in the auto transplanted neovagina. Gastroenterology.1991;100:1749-52. 
19. Toolenaar TA, Freundt I, Huikeshoven FJ, Drogendijk AC, Jeekel H, Chadha AS. The occurrence of diversion colitis in patients with a sigmoid neovagina. Human Pathol 1993;24:846-9.

20. Fotopoulou C, Neumann U, Klapp C, Lichtenegger W, Sehouli J. Long-term effects of neovaginal reconstruction with sigmoid loop technique on sexual function and self-image in patients with gynecologic malignancies: results of a prospective study. Gynecol Oncol. 2008;111(3):400-6.
21. Bean EJ, Mazur T, and Robinson AD. MayerRokitansky-Kuster-Hauser Syndrome: Sexuality, Psychological Effects, and Quality of Life. J Pediatr Adolesc Gynecol. (2009);22:339e346.

Cite this article as: Pan P. Surgical and functional outcome of sigmoid colon-vaginoplasty in MayerRokitansky-Kuster-Hauser syndrome. Int J Reprod Contracept Obstet Gynecol 2017;6:4441-6. 